\title{
Exámenes prequirúrgicos innecesarios y su costo en un hospital de Lambayeque
}

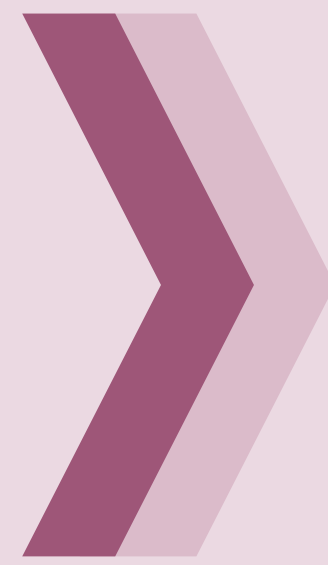

\author{
Unnecessary preoperative test \\ and the cost in a hospital of \\ Lambayeque
}

\section{Franco León-Jiménez, ${ }^{1}$ Edith Florián-Romero, ${ }^{2}$ Yvette Caján-Lontop, ${ }^{2}$ Luz Ventura-Sandoval, Marco Flores-Tucto ${ }^{3}$ y Angie Gastelo-Dávila ${ }^{2}$}

1. Médico internista. Docente de la Escuela de Medicina Humana de la Universidad Católica Santo Toribio de Mogrovejo. Chiclayo, Perú

2. Estudiante de la Escuela de Medicina Humana, Universidad Católica Santo Toribio de Mogrovejo. Chiclayo, Perú.

3. Médico cirujano. Docente de la Escuela de Medicina Humana de la Universidad Católica Santo Toribio de Mogrovejo. Chiclayo, Perú.

\section{RESUMEN}

INTRODUCCIÓN. En la práctica diaria antes de una cirugía suelen solicitarse exámenes preoperatorios sin ninguna utilidad. Sin embargo, las comorbilidades y el examen físico son los determinantes del desenlace en el acto quirúrgico. Овлетіvos. Determinar la frecuencia de exámenes prequirúrgicos innecesarios y su costo en pacientes con cirugía electiva del Hospital Regional Lambayeque de octubre de 2012 a julio de 2013. Material y método. Estudio descriptivo trasversal; muestreo aleatorio simple. Se compararon los exámenes solicitados con los recomendados por la guía de práctica clínica del National Institute for Health and Care Excellence (NICE) del 2003: Preoperative tests: The Use of routine preoperative tests for elective surgery. Resultados. Se revisaron 96 historias clínicas de pacientes con las siguientes cirugías: 62 colecistectomías $(64,5 \%), 16$ hernioplastias inguinales (16,67\%), 12 facoemulsificaciones (12,5\%), 5 cirugías de fractura expuesta $(5,21 \%)$, y 1 hernioplastia abdominal (1,04\%); se hallaron 1214 exámenes preoperatorios, 839 (69,1 \%) fueron innecesarios. Los más frecuentes fueron: riesgo quirúrgico (innecesario en 92,2 \%), tiempo de sangría (innecesario en $92 \%$ ) y grupofactor (innecesario en $89,8 \%$ ). Los exámenes más costosos fueron el antígeno de superficie para hepatitis B (S/.1428) y riesgo quirúrgico (S/.1185). El costo total de los exámenes innecesarios fue S/. 8858 (S/. 10630 aproximadamente en un año). Conclusión. En el Hospital Regional Lambayeque de octubre de 2012 a julio de 2013, hubo una elevada frecuencia de exámenes prequirúrgicos innecesarios y un alto costo asociado a los mismos. Los más costosos fueron antígeno de superficie para hepatitis $B$ y el riesgo quirúrgico.

Palabras clave. Costos y análisis de costos, período preoperatorio, cuidados preoperatorios, control de riesgo.

\begin{abstract}
INTRODUCTION. In daily practice, before surgery, preoperative tests often ordered without any utility. Comorbidities and physical examination, ancillary tests, are the determinants of outcome patient during surgery. OBJECTIVEs. To determine the frequency of unnecessary preoperative test and cost in patients with elective surgery in the Hospital Regional Lambayeque from October 2012 to July 2013. MATERIAL AND METHOD. It's a transversal descriptive study; simple random sampling. Requested tests were compared with those recommended by the clinical practice guideline of the National Institute for Health and Care Excellence (NICE) 2003: Preoperative tests: The use of preoperative routine tests for elective surgery. Results. The clinical histories of 96 patients were reviewed and show the next results: 62 cholecystectomies (64,5\%), inguinal hernia repair 16 (16,67\%), 12 phacoemulsification (12,5\%), 5 surgeries open fracture $(5,21 \%)$ and one abdominal hernia repair (1,04\%). Were found 1214 preoperative test, 839 (69,1\%) were unnecessary, being the most common surgical risk (in $92,2 \%$ of patients was unnecessary), bleeding time ( $92 \%$ ) and group and factor (unnecessary in $89,8 \%$ ). The test costliest was serological test for hepatitis B (S/. 1 428) and surgical risk (S/. 1185). The total cost of unnecessary preoperative tests from October 2012 to July 2013 was S/. 8858 (S/. 10630 about 1 year). Conclusion. In Hospital Regional Lambayeque from October 2012 to July 2013, there was a high frequency of unnecessary preoperative tests in elective surgeries and high costs associated with them. The tests that contributed more to the total cost were surface antigen for hepatitis $B$ and surgical risk.
\end{abstract}

KEY woRDs. Costs and cost analysis, preoperative period, preoperative care, routine diagnostic tests, risk management. 


\section{INTRODUCCIÓN}

Los exámenes preoperatorios son una práctica muy difundida en el quehacer médico; sin embargo, la mayoría de veces, su uso no se basa en la mejor evidencia disponible. Un examen prequirúrgico innecesario es un procedimiento realizado a un paciente no recomendado por una guía de práctica clínica, con resultados normales 0 en el que el hallazgo de alguna anormalidad no conlleva a ningún cambio en el manejo quirúrgico; su beneficio es pues, nulo. ${ }^{1}$

Una adecuada historia clínica y un detallado examen físico suelen ser suficientes para disminuir hasta en $60 \%$ la cantidad de exámenes preoperatorios de rutina. ${ }^{2}$ Si solo se solicitaran exámenes prequirúrgicos necesarios, disminuirían los costos por paciente, se descongestionarían los servicios de laboratorio y se reducirían los tiempos preoperatorios. ${ }^{3}$

A pesar de que las pruebas habitualmente realizadas en la valoración preoperatoria son de bajo costo, debido a su frecuente solicitud, pueden suponer una importante carga económica (costos directos). Esta carga, sumada a la carga social en jornadas laborales perdidas, tiempo e incomodidades para los pacientes, puede originar costos indirectos e intangibles considerables. ${ }^{4}$

Ibarra ${ }^{3}$ describe que es muy escasa la evidencia que apoye la utilización rutinaria de radiografías de tórax, electrocardiogramas, glucemias en ayunas, exámenes de orina y pruebas de coagulación en cirugías de bajo riesgo; no solicitar estas pruebas representaría una medida costo eficaz.

En Brasil, en el año 2013, en un estudio descriptivo transversal, se encontró que en 800 pacientes se realizaron un total de 3646 exámenes preoperatorios, de los cuales solo 82 (2,25\%) tenían alguna alteración en el resultado, pero generaron un cambio de conducta solo en $0,38 \%$ de estos. ${ }^{5}$

Exámenes prequirúrgicos solicitados de forma indiscriminada y no justificados por la sospecha de enfermedad producen un gran número de resultados anormales que en algunos casos obliga a repetir las pruebas 0 a solicitar otras distintas más agresivas, que pueden ocasionar un riesgo para el paciente, además de aumentar el grado de confusión y ansiedad del mismo. ${ }^{6}$
Ante la falta de información local, el objetivo del estudio fue determinar la frecuencia de exámenes prequirúrgicos innecesarios y su costo en pacientes con cirugía electiva menor del Hospital Regional Lambayeque, de octubre de 2012 a julio de 2013.

\section{MATERIAL Y MÉTODOS}

Estudio descriptivo trasversal ejecutado en el Hospital Regional Lambayeque de Chiclayo, hospital nivel III 1 del Ministerio de Salud. Mediante muestreo aleatorio simple, se eligieron 96 historias clínicas (de un total de 378) de pacientes atendidos por consulta externa, sin comorbilidades, e intervenidos por cirugía electiva de octubre de 2012 a julio de 2013, en los servicios de Traumatología, Oftalmología y Cirugía general. Se consultó a un cirujano general, un traumatólogo y a un oftalmólogo cuales eran las cirugías más frecuentes de su especialidad en este hospital. Se compararon los exámenes solicitados con los recomendados por la guía de práctica clínica del National Institute for Health and Care Excellence (NICE) del 2003: Preoperative tests: the use of routine preoperative tests for elective surgery.

El proyecto fue aprobado por el Comité de Ética e Investigación de la Facultad de Medicina de la Universidad Católica Santo Toribio de Mogrovejo y por la Dirección de investigación del Hospital Regional.

\section{RESULTADOS}

La edad promedio de los pacientes fue $52 \pm 17,6$ años, con un rango de 18 a 88 años; 73 pacientes eran mayores de 40 años (76\%); 55, mujeres $(57 \%)$ y 41 , hombres (43\%). Entre los varones, el promedio de edad fue 53 años y entre las mujeres, 57 años Se incluyeron 62 colecistectomías $(64,6 \%), 16$ hernioplastias inguinales $(16,7 \%)$, 12 facoemulsificaciones $(12,5 \%), 5$ limpiezas quirúrgicas de fractura expuestas $(5,2 \%)$ y 1 hernioplastia abdominal (1,04 \%). Tabla 1.

Se solicitaron 1214 exámenes prequirúrgicos, un promedio de $13 \pm 2$ por paciente (rango 7-20), de los cuales $839(69 \%)$ fueron innecesarios. En las 96 historias (100\%) se halló al menos un examen prequirúrgico inútil. El promedio de exámenes innecesarios por paciente fue de $8 \pm 2,9$. Los 


\section{Anta Méálica Pernana}

Tabla 1. Exámenes solicitados, los innecesarios y su costo según servicio: frecuencia de exámenes prequirúrgicos innecesarios y su costo en pacientes intervenidos por cirugía más frecuente en el Hospital Regional Lambayeque de octubre de 2012 a octubre de 2013

\begin{tabular}{|c|c|c|c|c|c|c|c|}
\hline \multirow[t]{2}{*}{ Servicio } & \multicolumn{2}{|c|}{ Historias clínicas } & \multicolumn{2}{|c|}{ Prequirúrgicos solicitados } & \multicolumn{2}{|c|}{ Prequirúrgicos innecesarios } & \multirow[t]{2}{*}{ Costo $(\mathrm{S} /)}$. \\
\hline & $\mathrm{N}$ & $(\%)$ & $\mathrm{N}$ & $(\%)$ & $\mathrm{N}$ & $(\%)$ & \\
\hline$\triangle$ Cirugía general & 79 & $(82,3)$ & 1019 & $(84,0)$ & 729 & $(86,8)$ & 5592 \\
\hline$\triangle$ Oftalmología & 12 & $(12,5)$ & 159 & $(13,0)$ & 79 & $(9,5)$ & 530 \\
\hline A Traumatología & 5 & $(5,2)$ & 36 & $(3,0)$ & 31 & $(3,7)$ & 125 \\
\hline Total & 96 & $(100,0)$ & 1214 & $(100,0)$ & 839 & $(100,0)$ & 6247 \\
\hline
\end{tabular}

exámenes prequirúrgicos innecesarios más solicitados fueron riesgo quirúrgico (en $92,2 \%$ de los pacientes), tiempo de sangría (92\%) y grupo sanguíneo y factor Rh (89,8\%). Tabla 2.

Los exámenes prequirúrgicos innecesarios que contribuyeron más al costo total fueron el antígeno de superficie para hepatitis $B(S / .1428)$ y riesgo quirúrgico (S/. 1185).

Solo un paciente $(0,01 \%)$ tenía seguro integral de salud (SIS). Tabla 3.

El costo total de los exámenes preoperatorios innecesarios en los tres servicios fue S/. 8858 (unos S/.10630 en un año); sin embargo, estos datos se aplican a las 96 historias clínicas analizadas. $\mathrm{Si}$ se considera el número total de historias clínicas (378), el costo promedio de exámenes prequirúrgicos innecesarios en un año para estas cinco cirugías fue aproximadamente S/. 41856.

\section{DISCUSIÓN}

Lamentablemente, los exámenes prequirúrgicos se siguen pidiendo de manera rutinaria, lo que puede perjudicar a los pacientes por un 'tratamiento excesivo' y aumenta los costos sin reducir las complicaciones pre y postoperatorias significativamente. ${ }^{3}$

Kaplan, ${ }^{8}$ en un análisis retrospectivo realizado en EE UU, encontró que de los resultados en 785 pruebas rutinarias de detección inicial en pacientes que serían sometidos a cirugía electiva, $60 \%$ no se justificaba.

La Sociedad Americana de Anestesiología (ASA) sugiere que la visualización de pruebas preoperatorias debe basarse en la información obtenida de los registros en la historia clínica, el examen físico, el tipo de paciente y en la extensión de la cirugía. ${ }^{?}$

Tabla 2. Exámenes prequirúrgicos más frecuentes y costosos: frecuencia de exámenes prequirúrgicos innecesarios y su costo en pacientes intervenidos por cirugía más frecuente en el Hospital Regional Lambayeque de octubre de 2012 a octubre de 2013

\begin{tabular}{|c|c|c|c|c|c|c|c|c|c|}
\hline \multirow[t]{2}{*}{ Servicio } & \multicolumn{2}{|c|}{ Riesgo quirúrgico } & \multicolumn{2}{|c|}{ Grupo y factor } & \multicolumn{2}{|c|}{ Tiempo de sangría } & \multicolumn{2}{|c|}{$\mathrm{HbAgs}^{\star}$} & \multirow{2}{*}{$\begin{array}{l}\text { Costo total en } \\
\text { el servicio (S/.) }\end{array}$} \\
\hline & $\mathrm{N}$ & $\%$ & $\mathrm{~N}$ & $\%$ & $\mathrm{~N}$ & $\%$ & $\mathrm{~N}$ & $\%$ & \\
\hline$\Delta$ Cirugía general & 70 & 84,4 & 66 & 83,5 & 66 & 82,5 & 45 & 90,0 & 5592,50 \\
\hline A Traumatología & 3 & 3,6 & 3 & 3,8 & 3 & 3,7 & - & - & 125,00 \\
\hline$\triangle$ Oftalmología & 10 & 12,0 & 10 & 12,7 & 11 & 13,8 & 5 & 10,0 & 530,40 \\
\hline Total & 83 & 100,0 & 79 & 100,0 & 80 & 100 & 50 & 100,0 & 6247,90 \\
\hline
\end{tabular}

HbAgs: antígeno de superficie. 
Tabla 3. Exámenes prequirúrgicos innecesarios más frecuentes: total de exámenes solicitados, costo unitario y su contribución al costo total

Examen preoperatorio

$\triangle$ Serología para hepatitis $B$

$\triangle$ Riesgo quirúrgico

$\triangle$ Perfil hepático

$\triangle$ Serología para VIH

$\triangle$ Electrocardiograma
Total de exámenes pedidos

Costo por examen $(\mathrm{S} /$.

Total (S/.)

$\begin{array}{rrr}50 & 28,6 & 1430,0 \\ 83 & 14,3 & 1186,9 \\ 41 & 24,3 & 996,3 \\ 58 & 12,5 & 725,0 \\ 62 & 7,2 & 446,4\end{array}$

El Colegio Americano de Médicos (ACP, del inglés American College of Physicians) recomienda que las pruebas de laboratorio se deberían ordenar con criterios selectivos y restrictivos, siempre con el apoyo de justificación clínica. El descubrimiento de los cambios en las pruebas que se aplican a pacientes clínicamente sanos en general no influyen en el tratamiento y, por lo general, no afectan el plan de trabajo en el período perioperatorio. ${ }^{9}$

El Hospital Regional Lambayeque debería evaluar el costo-beneficio de los exámenes prequirúrgicos en pacientes asintomáticos. En el presente estudio se ha demostrado que se podría ahorrar mucho dinero si se usaran los protocolos correctamente. Así, el dinero ahorrado podría dirigirse a mejorar la calidad del servicio en salud y los pacientes tendrían más accesibilidad a este servicio.

La indicación de radiografía de tórax en forma rutinaria no está apoyada por evidencias que demuestren su utilidad, pues solo $2 \%$ de las radiografías realizadas implican un cambio en el manejo de los pacientes, lo que sugiere un alto costo e inconvenientes de orden laboral, personal y de infraestructura, con un beneficio potencial muy limitado. ${ }^{10-13}$ Por lo que se sostiene no realizarla en pacientes asintomáticos respiratorios, y se sugiere su indicación en los pacientes con patología respiratoria, patología cardiovascular, fumadores de más de 20 cigarrillos por día, obesos con índice de masa corporal mayor de $30 \mathrm{y} / 0$ pacientes con antecedentes de cáncer y riesgo de metástasis. ${ }^{10-12,14}$

El electrocardiograma solo debería ser solicitado cuando exista indicación clínica justificada. Esta práctica médica elimina costos innecesarios, sin comprometer la seguridad y la calidad de atención de los pacientes. Se sugiere que se realice a partir de la edad de 50 años en varones y de 60 años en mujeres. ${ }^{3,12,14}$ Sin embargo, otros autores plantean realizarlo en todo paciente mayor de 40 años sin estudios electrocardiográficos previos. ${ }^{6,14}$

Las pruebas de función hepática no se indican de rutina, solo en pacientes con historia reciente de enfermedad hepática o del tracto biliar. Por otro lado, las pruebas hematológicas se deben utilizar en casos de sangrados anormales. El tiempo de protrombina se indica en pacientes con leucemia o pacientes con disfunción hepática. ${ }^{14-16}$

Los estudios complementarios solicitados en una valoración preoperatoria están influenciados por las condiciones siguientes: la necesidad de reconocer patologías asintomáticas que puedan precisar un tratamiento prequirúrgico o un cambio en el manejo anestésico o quirúrgico del paciente; la existencia de una valoración preoperatoria organizada o consulta preoperatoria específica, para prevenir complicaciones posquirúrgicas, en atención a protocolos de actuación y programas de salud; la implementación de acciones de pesquisa en función de factores de riesgo identificada; la actitud defensiva de los profesionales ante posibles reclamos judiciales., 3

Los exámenes preoperatorios innecesarios aumentan los costos sin reducir las complicaciones pre y postoperatorias. Por lo tanto, es fundamental el uso de guías de práctica clínica adaptadas a la realidad de cada centro hospitalario. La metodología para la adaptación de una guía internacional es bastante laboriosa y requiere la 
participación de varios especialistas (internistas, cirujanos, anestesiólogos, personal de enfermería, médicos de atención primaria, entre otros). La comunicación constante entre los diversos actores de este proceso es vital. Otra posibilidad es la generación de protocolos de manejo para estandarizar los criterios a utilizar.

El uso indiscriminado de estudios complementarios prequirúrgicos, sin tener en cuenta el perfil individual del sujeto, sin medir la efectividad esperada para disminuir los riesgos anestésicos, de supresión del dolor y/o de las complicaciones

\section{REFERENCIAS BIBLIOGRÁFICAS}

I. Garcia A, Caballé I, GiménezA. En: Imma Caballe M. Uso adecuado del laboratorio clínico. Barcelona: Elsevier-Masson; 2007.

2. Turnbull JM, Buck C. The value of preoperative screening investigations in otherwise healthy individuals. Arch Intern Med. 1987; |47:| I0I-1 I05.

3. Ibarra P. ¿Cuáles exámenes de laboratorio preanestésicos se necesitan en pacientes asintomáticos? Rev Colomb Anestesiol. 2007;35(4):30I-3I2.

4. Ajimura FY, Maia ASSF, Hachlya A, Watanabe AS. Preoperative laboratory evaluation of patients aged over 40 years undergoing elective non cardiac surgery. São Paulo Med J. 2005; 123:50-53.

5. De Sousa Soares D, Ribeiro Marques Brandão R, Nogueira Mourão MR, Fernandes de Azevedo VR, Vieira Figueiredo A, Santana Trindade E. Relevancia de los exámenes de rutina en pacientes de bajo riesgo sometidos a cirugías menores. Rev Bras Anestesiol. 2013;63(2):197-20I.

6. Sánchez G. Recomendaciones para la solicitud de estudios complementarios en la cirugía electiva.Argentina:Hospital Olvarria; 2005 [accesado el 3 de mayo de 20l4]. Disponible en: http://www. hospitalolavarria.com.ar/trabajos\%5Ccirugia_recomendaciones_ para_la_solicitud_de_estudios_complementarios.htm

7. National Institute for Health and Care Excellence (NICE). Preoperative tests (CG3): The use of routine preoperative tests for elective surgery. United Kingdom: NICE; 2003. [accesado el 3 de mayo de 2014]. Disponible en: http://publications.nice.org.uk/ preoperative-tests-cg3

8. Kaplan E. The usefulness of preoperative laboratory screening. JAMA. 1985;253:3576.

9. American Society of Anesthesiologists Task Force on Preanesthesia Evaluation: Practice advisory for preanesthesia postoperatorias esperadas, ha hecho que una masa de recursos económicos sean destinados en forma constante a fines que no parecen ser los adecuados.

El presente estudio solo consideró cuatro servicios (Traumatología, Oftalmología y Cirugía General), de los cuales se analizaron las cinco operaciones más frecuentes (colecistectomías, hernioplastias inguinales, facoemulsificaciones, limpiezas quirúrgicas de fractura expuestas, hernioplastia abdominal), sin embargo, es probable que los costos deben ser altos también en otros servicios. evaluation: An updated report by the American Society of Anesthesiologists TaskForce on Preanesthesia Evaluation. Anesthesiology. 2012;1 16:522-538.

10. García-Miguel FJ, García Caballero J, Gómez de Caso-Canto JA. Indicaciones de la radiografía de tórax para la valoración preoperatoria en cirugía programada. Rev Esp Anestesiol Reanim. 2002;49:80-88.

11. Johnson RK. Mortimer AJ. Routine pre-operative blood testing: is it necessary? Anaesthesia. 2002;57:914-917.

12. García-Miguel FJ, García Caballero J, Gómez de Caso-Canto JA. Indications for electrocardiogram in the preoperative assessment for programmed surgery. Rev Esp Anestesiol Reanim. 2002;49:5-12.

13. Vilarasau Farré J, Martín-Baranera M, Oliva G. Encuesta sobre la valoración preoperatoria en los centros quirúrgicos catalanes (I). ¿Cuál es la práctica preoperatoria? Rev Esp Anestesiol Reanim. 200I;48(I):4-10.

14. Contreras M. Evaluación médica preoperatoria como predictor de riesgo quirúrgico y no quirúrgico. Comunidad y Salud [revista en Internet].20 I0;8(I):83-96. [accesado el I 6 de febrero de 20 I4]. Disponible en: http://www.scielo.org.ve/scielo.php?script=sci_ arttext\&pid=S|690-329320 I0000 I000 I I \&Ing=es.

15. Sociedad Argentina de Cardiología. Consenso de evaluación del riesgo cardiovascular en cirugía no cardiaca. Rev Argent Cardiol. 2005;73(5):38I-396.

16. Rojas-Rivera W. Evaluación de los exámenes preoperatorios. Acta Méd Costarric [revista en Internet]. 2006;48(4):208-2II. [accesado el 16 de febrero de 2014]. Disponible en: http:// www.scielo.sa.cr/scielo.php?script=sci_arttext\&pid=S000 I $60022006000400013 \&$ Ing=es.

\section{Correspondencia}

Dr. Franco Ernesto León-Jiménez

francoernestole@gmail.com

Fecha de recepción: 10 de abril de 2014

Fecha de aceptación: 19 de mayo de 2014

\section{Conflictos de interés}

Los autores declaran no tener conflictos de interés durante el planteamiento, ejecución de la investigación y la elaboración del artículo para su publicación. 